\title{
Zur Kenntniss einiger Nitroverbindungen der Pyridinreihe
}

\author{
von \\ H. Weidel und E. Murmann.
}

Aus dem I. chemischen Laboratorium der k. k. Universität in Wien.

(Vorgelegt in der Sitzung am 11. Juli 1895.)

Bisher hat man sich vergebens bemüht, das Pyridin oder die Homologen desselben zu nitriren. Decker ${ }^{1}$ erklärt dies durch den besonderen Einfluss des tertiären Stickstoffs, der wie ein stark negativer Rest wirkt und eine Substitution der $\mathrm{NO}_{2}$-Gruppe in der sauren Lösung verhindert. Auch Marckwald ${ }^{2}$ sprach die Ansicht aus, dass der tertiäre Stickstoff eine acidificirende Wirkung ausübt und den Eintritt der Nitrogruppe behindert. Er konnte thatsächlich Pyridinderivate, in welchen durch den Eintritt der $\mathrm{COOH}$ - und $\mathrm{NH}_{2}$-Gruppe der Einfluss des Stickstoffs abgeschwächt war, durch Behandlung mit Salpetersäure in Nitroverbindungen überführen. So hat er aus der $\alpha^{\prime}$-Oxynicotinsäure die $\beta^{\prime}$-Nitro- $\alpha^{\prime}$-Oxynicotinsäure hergestellt. Wie hier die Carboxyl- und Hydroxylgruppe von bestimmendem Einfluss auf den Verlauf der Reaction war, ebenso ist dies auch der Fall bei der $\alpha^{\prime}$-Amidonicotinsäure, aus welcher er die $\beta$-Nitro- $\alpha^{\prime}$-Amidonicotinsäure hergestellt hat. Marckwald vermuthet aus den früher angegebenen Gründen, dass die Oxypyridine leichter einer Nitrirung zugänglich sein werden. Dies ist, wie in den folgenden Blättern gezeigt werden soll, auch thatsächlich der Fall.

\footnotetext{
1 Journ. f. pr. Chem. [2], 45, 52.

9 Berl. Ber., 26, 2187 und 27, 1317.
} 
Wir haben vor einigen Jahren eine Untersuchung begonnen, die darauf abzielte, Nitroverbindungen der Pyridinderivate herzustellen. Nach dem Erscheinen der Marckwald'schen Abhandlungen haben wir unseren Versuchen keine weitere Ausdehnung gegeben und wir wollen nur über die beim $\beta$-Oxypyridin gewonnenen Erfahrungen berichten.

Die Darstellung des $\beta$-Oxypyridins ist bisher mit nicht sehr günstigen Resultaten durchgeführt worden, weil die Bildung der hiezu erforderlichen $\beta$-Sulfonsäure, wie $O$. Fischer und Renouf ${ }^{1}$ selbst angeben, in quantitativer Hinsicht sehr ungünstig verläuft. Es werden bei der Sulfonirung des Pyridins bestenfalls $30 \%$ der theoretischen Ausbeute erhalten. Wir haben zunächst getrachtet, bessere Ausbeuten zu erzielen und haben versucht, die Einwirkung der Schwefelsäure bei einer höheren Temperatur, als Fischer angibt, vorzunehmen. Dabei beobachteten wir, dass dieses Moment nicht von besonderem Einfluss auf den Verlauf der Reaction ist. Um grössere Quantitäten des Gemisches von Pyridin und Schwefelsäure auf Temperaturen zu bringen, die über $300^{\circ}$ liegen, haben wir in die Masse schwefelsaure Salze, wie Kali-, Natronsulfat etc. eingetragen. Die Ausbeute wurde dadurch nicht verbessert und schwankte zwischen 25 und $30 \%$.

Bekanntlich verlaufen die Chlorungen von organischen Verbindungen in vielen Fällen günstiger, wenn in die zu substituirenden Substanzen Metallchloride, wie Aluminiumchlorid, Eisenchlorid etc. eingetragen werden. Wir haben nun nach einer Verbindung gesucht, die ähnliche Wirkung für die Einführung des Schwefelsäurerestes hat und haben gefunden, dass eine Reihe von Sulfaten die Bildung der Sulfonsäure durch ihre Gegenwart wesentlich erleichtern. Unter diesen hat sich das Aluminiumsulfat als das tauglichste erwiesen.

Dass das Thonerdesulfat als Schwefelsäureüberträger ähnlich dem Chloraluminium bei den Chlorungen wirkt und nicht etwa die höhere Temperatur die Ursache ist, zeigt die Thatsache, dass andere Metallsulfate, durch deren Zugabe man ja dieselbe hohe Temperatur erreichen kann, die Bildung der

1 Berl. Ber. 17, 763. 
Sulfonsäure in quantitativer Hinsicht nicht beeinflussen. Durch die Sulfonirung bei Gegenwart von Aluminiumsulfat konnten wir 60\% des Pyridins in die Pyridin- $\beta$-Sulfonsäure verwandeln, und zwar haben wir die folgenden Verhältnisse eingehalten. Ein Gemisch von $250 \mathrm{~g}$ Pyridin, $1000 \mathrm{~g}$ Schwefelsäure (hergestellt aus $750 \mathrm{~g}$ englischer Schwefelsäure und $250 \mathrm{~g}$ Schwefelsäureanhydrid) und $100 \mathrm{~g}$ entwässertes Aluminiumsulfat wurden in einem grossen Kolben im Sandbade auf $330^{\circ}$ bis $350^{\circ} \mathrm{C}$. durch $40-60$ Stunden erhalten. Während dieser Zeit wird die verdampfte Schwefelsäure von Zeit zu Zeit ersetzt. Das Aluminiumsulfat löst sich allmälig in der Masse auf; dabei entwickelt sich etwas schwefelige Säure und falls $z u$ grosse Quantitäten der Säure abdunsten, auch Pyridin. ${ }^{1}$ Nach der angegebenen Zeit hat sich fast die ganze Menge des Sulfates gelöst und der Kolbeninhalt ist in ein dickliches, braungelbgefärbtes Product verwandelt. Dieses wird in einer grossen Quantität Wasser gelöst, mit Ätzbaryt alkalisch gemacht, vom Bariumsulfat filtrirt, nach der Entfernung des unverbrauchten Pyridins durch Destillation wird die Flüssigkeit durch Einleiten von Kohlensäure in der Siedehitze vom überschüssigen Baryt befreit. Dabei fällt auch die in Lösung gegangene Thonerde aus. Die von der Ausscheidung filtrirte Lösung wird concentrirt und hierauf mit Ammoniumcarbonat versetzt. Dadurch wird pyridin- $\beta$-sulfonsaures Ammon gebildet. Der ausfallende kohlensaure Baryt hat uns zur quantitativen Bestimmung der Ausbeute gedient. Anderseits haben wir uns über dieselbe durch Bestimmung der Mengen von $\beta$-Oxypyridin, welches aus einem aliquoten Theile des Ammonsalzes hergestellt wurde, orientirt. Bei Einhaltung der gegebenen Verhältnisse erzielten wir eine Ausbeute bis $z$ u $65 \%$. Bei Wiederholung der Versuche kamen wir zu ähnlichen Werthen und wir fanden, dass im ungünstigsten Falle etwa $45-50 \%$ des angewendeten Pyridins in die Sulfonsäure verwandelt wurde.

Die Sulfate des Magnesiums, Zinks, Chroms, Ammoniaks üben diese Ausbeute vergrössernde Wirkung nicht aus. Ferri-

1 Bei den Operationen, bei welchen Pyridin auftrat, war in der Regel die Ausbeute niedriger. 
sulfat verhindert geradezu die Bildung der Sulfonsäure. Selbstverständlich wurde die Temperatur auch bei Anwendung dieser Sulfate auf circa $340^{\circ}$ erhalten.

Die folgende Zusammenstellung soll die Verhältnisse betreffs der Ausbeute an Pyridinsulfonsäure bei Verivendung verschiedenartiger Sulfate besonders illustriren.

\begin{tabular}{|c|c|c|}
\hline \multicolumn{2}{|c|}{ Auf $50 \mathrm{~g}$ Pyridin und $200 \mathrm{~g} \mathrm{SH}_{2} \mathrm{O}_{4}$} & \multirow{2}{*}{$\begin{array}{c}\text { Ausbeute an } \\
\text { Sulfonsäure in } \\
0 / \%\end{array}$} \\
\hline & $\begin{array}{c}\text { Menge der } \\
\text { zugegebenen } \\
\text { Sulfate in } g\end{array}$ & \\
\hline \multirow{2}{*}{$\left.\mathrm{K}_{2} \mathrm{SO}_{4} \cdot \ldots \ldots \ldots\right\}$} & 20 & 18 \\
\hline & 50 & $4 \tilde{5}$ \\
\hline \multirow{2}{*}{$\left.\mathrm{Mg} \mathrm{SO}_{4} \ldots \ldots \ldots\right\}$} & 20 & 28 \\
\hline & 50 & 34 \\
\hline \multirow{2}{*}{$\left.\mathrm{Al}_{2}\left(\mathrm{SO}_{4}\right)_{3} \ldots \ldots \ldots\right\}$} & 20 & 29 \\
\hline & 50 & 60 \\
\hline $\mathrm{Cr}_{2}\left(\mathrm{SO}_{4}\right)_{3} \ldots \ldots \ldots$ & 50 & 30 \\
\hline \multirow{2}{*}{$\mathrm{Fe}_{2}\left(\mathrm{SO}_{4}\right)_{3} \ldots \ldots \ldots$} & 20 & 5 \\
\hline & 50 & 6 \\
\hline
\end{tabular}

Diese nach dem angegebenen Verfahren gewonnene Sulfosäure ist, wie zu erwarten war, identisch mit der von $\mathrm{O}$. Fischer beschriebenen, was daraus hervorgeht, dass sie bei Einwirkung von $\ddot{A}$ tzkali $\beta$-Oxypyridin liefert.

Das Ammonsalz, welches man als Rohproduct gewinnt, ist fast rein und zeichnet sich durch eine ausserordentliche Kry. stallisationsfähigkeit aus. Beim freiwilligen Verdunsten einer Lösung dieses Salzes bilden sich nicht selten dicke, monocline Prismen, die eine beträchtliche Grösse erreichen. Herr Hofrath v. Lang hat die Liebenswürdigkeit gehabt, dieses Salz einer krystallographischen Untersuchung zu unterziehen und theilt uns über seine Beobachtungen Folgendes mit:

"Krystallsystem: monoclinisch.

$a: b: c=1 \cdot 5866: 1: 2 \cdot 2897$. 
$a c=101^{\circ} 42^{\prime}$.

Beobachtete Flächen: (001), (100), (110), (101).

Die Krystalle sind nach der Fläche 100 sehr gut spaltbar, weniger gut nach der Fläche 001 «.

Das Ammonsalz ist krystallwasserfrei. Die Analye desselben zeigt, dass das Salz nach der Formel $\mathrm{C}_{5} \mathrm{H}_{4} \mathrm{SO}_{3}\left(\mathrm{NH}_{4}\right) \mathrm{N}$ zusammengesetzt ist.

$0.2490 \mathrm{~g}$ Substanz gaben $0.3143 \mathrm{~g}$ Kohlensaure und $0.0949 \mathrm{~g}$ Wasser. II. $0 \cdot 2929 \mathrm{~g}$ Substanz gaben $0.3962 \mathrm{~g}$ Bariumsulfat.

III. $0.207 \mathrm{~g} \mathrm{~g}$ Substanz gaben $29.5 \mathrm{~cm}^{3} \mathrm{~N}$ bei $750 \mathrm{~mm}$ und $17^{\circ} \mathrm{C}$.

In 100 Theilen:

\begin{tabular}{ccccc}
\multicolumn{2}{c}{ I } & II & III & $\underbrace{\text { Berechnet }}$ \\
$\mathrm{C} \ldots \ldots \ldots \ldots$ & $34 \cdot 42$ & - & - & $34 \cdot 09$ \\
$\mathrm{H} \ldots \ldots \ldots \ldots$ & $4 \cdot 23$ & - & - & 4.54 \\
$\mathrm{~S} \ldots \ldots \ldots$ & - & $18 \cdot 57$ & - & 18.18 \\
$\mathrm{~N} \ldots \ldots \ldots$ & - & - & $16 \cdot 26$ & 15.93
\end{tabular}

Dieses Ammonsalz lässt sich mit Vortheil direct zur Darstellung des $\beta$-Oxypyridins verwenden. $\mathrm{Zu}$ diesem Ende bringt man dasselbe mit etwa der vierfachen Menge Ätzkali in eine geräumige Silberschale und erhitzt im Ölbade auf $170-178^{\circ} \mathrm{C}$. Die 'Temperatur muss genau eingehalten und durch ein in die Schmelze eingesenktes Thermometer bestimmt werden. Hat man $1-2$ Stunden auf die angegebene Temperatur erhitzt, so geht das Ammonsalz (welches zuerst das Ammon abgegeben hat) quantitativ in $\beta$-Oxypyridin über. Die Schmelze wird nun in Wasser gelöst, mit Salzsäure bis zur schwach sauren Reaction angesäuert und hierauf zur Trockene eingedampft. Der Trockenrückstand liefert beim Behandeln mit Alkohol eine Lösung, aus welcher nach dem Abdestilliren das salzsaure Oxypyridin sich ausscheidet. Diese Rohausscheidung wird direct durch Versetzen mit einer sehr concentrirten Lösung von kohlensaurem Natron zerlegt. Dabei wird das Oxypyridin seiner Schwerlöslichkeit wegen in kleinen, noch dunkelgefärbten Krystallen abgeschieden, ${ }^{1}$ die durch wiederholtes Umkrystallisiren (even-

1 Die abgesaugten, braungefärbten Laugen enthalten noch eine geringe Quantitat von Oxypyridin, die durch wiederholtes Ausschütteln mit Äther gewonnen werden kann. 
tuell Entfärben) sehr leicht und ohne Verlust zu reinigen sind. Nach diesem Verfahren erhält man nahezu theoretische Ausbeuten an Oxypyridin. Dasselbe zeigt alle Eigenschaften, die Fischer und Renouf angaben, und besitzt den Schmelzpunkt von $129^{\circ}$.

Das $\beta$-Oxypyridin haben wir auf rerschiedene Weisen $z u$ nitriren versucht. Als wir die Lösung desselben in concentrirter Schwefelsäure mit Salpetersäure behandelten, beobachteten wir den Eintritt einer stürmischen Reaction und fanden, dass in der Masse sehr kleine Quantitäten eines gelben, krystallinischen Körpers gebildet waren, die offenbar ein Nitroproduct des Oxypyridins darstellen. Die Hauptmenge des Pyridons wird jedoch bei dieser Einwirkung total verbrannt und es fanden sich grosse Quantitäten von Oxalsäure vor. Ebenso heftig verläuft die Reaction, wenn man rothe, rauchende oder selbst gewöhnliche Salpetersäure aufs Oxypyridin einwirken lässt. Auch dabei werden grosse Mengen von Oxalsäure gebildet, während das Nitroproduct nur spurenweise entsteht. Wir haben daher versucht, die Acetylverbindung des $\beta$-Oxypyridins zu nitriren, aber auch diese Substanz wird durch Salpetersäure zerstört. Mit günstigem Erfolge jedoch konnten wir die Nitrirung durchführen, als wir das Acetyloxypyridin in Essigsäureanhydrid lösten und die Lösung allmälig mit Salpetersäure versetzten, die vorher mit salpetriger Säure gesättigt war. Die Lösung des Acetylproductes muss gut gekühlt werden, da jeder Tropfen der einfallenden Säure eine lebhafte Reaction hervorbringt. Es empfiehlt sich, dem Gewichte nach nicht mehr Salpetersäure einzutragen, als Acetylproduct verwendet wurde. Nimmt man nun den Kolben aus dem Eiswasser und lässt ihn gerröhnliche Temperatur annehmen, so tritt noch eine Nachreaction ein, die durch vorsichtiges Erwärmen zu Ende geführt wird. Die Lösung destillirt man hierauf im Vacuum ab, wodurch eine dunkelgelb gefärbte, zähe Masse, die etwas unzersetztes Acetyloxypyridin enthält, erhalten wird. Oxalsäure ist dabei nicht entstanden. Die acetylirten Nitroproducte sind nur schwierig zum Krystallisiren zu bringen und desswegen wird der Destillationsrückstand direct mit Natronlauge behandelt, wodurch Zerlegung der 
Acetylproducte eintritt. So wie Lösung eingetreten ist, lässt man erkalten und neutralisirt die Flüssigkeit durch Zugabe von Salzsäure. Nach einiger Zeit beginnt in der eingeengten Flüssigkeit reichliche Ausscheidung von gelben oder gelbroth gefärbten Krystallen, die ein Gemenge mehrerer Substanzen darstellen. Das Hauptproduct ist ein Dinitro- $\beta$-Oxypyridin; neben demselben sind noch zwei Mononitro- $\beta$-Oxypyridine entstanden. Die Trennung und Reinigung dieserVerbindungen nimmt man in folgender Weise vor: Die abgesaugte Ausscheidung wird mit heissem Wasser behandelt, wodurch ein schwer löslicher Theil (a) entfernt wird. Die wässerige Lösung wird mit Äther wiederholt geschüttelt. Derselbe hinterlässt nach dem Verjagen ein gut krystallisirendes Product $(b)$, während aus der ausgeschüttelten Lösung bei entsprechender Concentration die Abscheidung einer krystallinischen Masse (c) stattfindet. Wir wollen zuerst die Aufarbeitung und Reinigung der Hauptmasse $(b)$ besprechen.

\section{Dinitrooxypyridin.}

Die Substanz (b) ist noch verunreinigt durch kleine Quantitäten $(c)$ und kann von diesen nur durch wiederholtes Umkrystallisiren aus Benzol getrennt werden. (c) wird von diesem Lösungsmittel sehr schwer aufgenommen. Das so gereinigte Product $(b)$, welches als Dinitro- $\beta$-Oxypyridin zu betrachten ist, wird dann in absolutem Äther gelöst. Die mit Thierkohle geschüttelte Lösung liefert beim langsamen Abdunsten ein lockeres Haufwerk von sattgelb gefärbten, ziemlich stark glänzenden Krystallnadeln, die auch von Wasser in der Wärme aufgenommen werden und sich aus diesem Lösungsmittel in haarfeinen, verfilzten Kryställchen abscheiden. Die Verbindung ist auch in Alkohol löslich. Beim Erhitzen schmilzt die Substanz bei $133^{\circ}$ (uncorr.). Für die Analyse wurde die Substanz bei $100^{\circ}$ zur Gewichtsconstanz gebracht und ergab Zahlen, aus welchen die Formel $\mathrm{C}_{5} \mathrm{H}_{2}\left(\mathrm{NO}_{2}\right)_{2} \mathrm{OH}$. N gerechnet wurde.

I. $0 \cdot 2401 g$ Substanz gaben $0.2832 \mathrm{~g}$ Kohlensäure und $0.0377 \mathrm{~g}$ Wasser.

II. $0.2524 \mathrm{~g}$ Substanz gaben $0.2985 \mathrm{~g}$ Kohlensäure und $0.0422 \mathrm{~g}$ Wasser.

III. $0 \cdot 1755 \mathrm{~g}$ Substanz gaben $35 \cdot 5 \mathrm{~cm}^{3}$ Stickstoff bei $757 \cdot 8 m u$ und $22.9 \mathrm{C}$. 
In 100 Theilen:

$\begin{array}{ccccc} & \text { I } & \text { II } & \text { III } & \underbrace{\text { Berechnet }} \\ \text { C } \ldots \ldots \ldots \ldots & 32 \cdot 16 & 32 \cdot 25 & - & 32 \cdot 43 \\ \text { H } \ldots \ldots \ldots & 1 \cdot 74 & 1 \cdot 85 & - & 1 \cdot 62 \\ \text { N } \ldots \ldots \ldots \ldots & - & - & 22 \cdot 73 & 22 \cdot 70\end{array}$.

Das Dinitro- $\beta$-Oxypyridin liefert mit Säuren Verbindungen, die äusserst zersetzlich sind und die aus diesem Grunde nicht in brauchbare Form gebracht werden konnten. Dagegen bildet die Substanz mit Alkalien, in welchen sie mit intensiv röthlichgelber Farbe löslich ist, beim Abdunsten krystallisirte Producte, in welchen ein Metallatom eingetreten ist. Die Natronverbindung stellt matte Krystallblättchen von hochgelber Farbe dar, die beim Erhitzen ziemlich kräftig explodiren und welche bei der Natriumbestimmung folgende Werthe lieferten:

$0 \cdot 4215 \mathrm{~g}$ Substanz gaben $0.1467 \mathrm{~g}$ Natriumsulfat.

In 100 Theilen:

$\mathrm{Na} \ldots \ldots+11 \cdot 27 \quad \mathrm{C}_{11 \cdot 11}^{\mathrm{C}_{5} \mathrm{H}_{2}\left(\mathrm{NO}_{2}\right)_{2} \mathrm{ONaN}}$

Die Lösung der Nitroverbindung in Ammoniak wird durch Silbernitrat gelbroth gefällt. Bei Einwirkung von Reductionsmitteln entfärbt sich die Lösung der Dinitroverbindung und es bildet sich, wenn etwa Zinn und Salzsäure verwendet wurde, nach der Entfernung des in Lösung gegangenen Metalls beim längeren Stehen der eingedampften Lösung eine krystallinische Ausscheidung, welche ausserordentlich veränderlich ist, sich bald braun färbt und in welcher Salmiakkrystalle enthalten sind. Bei der Reduction hat sich offenbar ein Amidoproduct gebildet. Dieses konnte seiner Zersetzlichkeit wegen nicht isolirt werden. Auch die anderen Reductionsmittel liefern keine besseren Resultate.

\section{Untersuchung von (c).}

Der mit $(c)$ bezeichnete Antheil ist unreines Mononitro$\beta$-Oxypyridin, welches seiner Schwerlöslichkeit wegen in Benzol und Äther von Dinitrooxypyridin durch wiederholtes Fxtrahiren mit diesen Lösungsmitteln völlig getrennt werden 
kann. Nach öfterem Umkrystallisiren aus Wasser (eventuell unter Anwendung von Thierkohle) wird die Substanz in lichtcitronengelben, kleinen, schwachglänzenden Krystalltafeln, die in dünnen Schichten fast farblos erscheinen, erhalten. Die Verbindung wird auch von Alkohol aufgenommen und scheidet sich aus demselben in Blättchen ab, die eine schwach citronengelbe Farbe besitzen. Die Substanz schmilzt bei $210-211^{\circ}$ (uncorr.). Das Mononitroproduct ist in verdünnten Alkalien mit gelber Farbe löslich. Auch diese Lösungen scheiden beim Eindampfen gelbgefärbte krystallinische Verbindungen ab. Die Analyse des krystallwasserfreien, bei $100^{\circ}$ getrockneten Productes ergab Werthe, welche mit den für Mononitrooxypyridine gerechneten in bester Übereinstimmung stehen.

I. $0 \cdot 2782 \mathrm{~g}$ Substanz gaben $0 \cdot 4382 \mathrm{~g}$ Kohlensäure und $0.0759 \mathrm{~g}$ Wasser. II. $0.2029 \mathrm{~g}$ Substanz gaben $35.1 \mathrm{~cm}^{3}$ Stickstoff bei $755 \mathrm{~mm}$ und $15^{\circ} \mathrm{C}$.

In 100 Theilen:

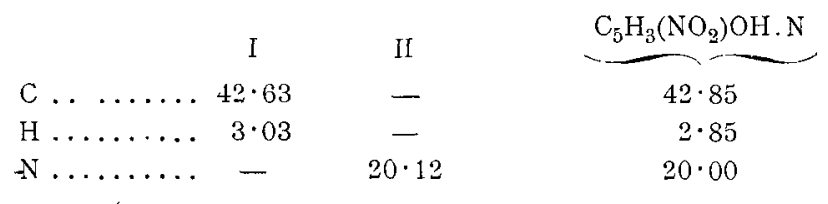

Das beschriebene Nitro- $\beta$-oxypyridin ist auch befähigt, mit Salzsäure eine Verbindung einzugehen; jedoch ist auch diese ziemlich zersetzlich.

Bei Reduction liefert (c) eine Amidoverbindung, die weniger veränderlich ist, als die des Dinitrooxypyridins. Da jedoch das Mononitrooxypyridin nur in untergeordneter Menge bei der Nitrirung des Oxypyridins entsteht, wir nur verhältnissmässig kleine Mengen zur Verfügung hatten, so konnten wir auf eine nähere Untersuchung desselben nicht eingehen.

\section{Untersuchung von $(a)$.}

(a) ist ebenfalls ein Mononitrooxypyridin, welches in Benzol und Äther vollständig unlöslich ist und nur sehr schwer von siedendem Wasser und Alkohol aufgenommen wird. Man reinigt die Verbindung dadurch, dass man sie in sehr ver- 
dünnter Lösung mit Thierkohle kocht und das Filtrat eindampft, die ausfallenden Krystalle dann aus grossen Mengen siedenden Alkohols umkrystallisirt. Auf diese Weise gewinnt man wollige, mattgelb gefärbte, glanzlose, haarförmige, lange Krystallnadeln, die in Alkalien mit intensiv blutrother Farbe löslich sind. Die Substanz, welche von dem vorher beschriebenen Nitroproduct schon durch die Löslichkeitsverhältnisse wesentlich unterschieden ist, zeigt auch einen viel höheren Schmelzpunkt. Beim Erhitzen wird sie bei circa $255^{\circ} \mathrm{C}$. dunkel und schmilzt erst bei $295-298^{\circ}$ C. (uncorr.) unter totaler Zersetzung. Die alkalischen Lösungen dieser Nitroproducte scheinen sich beim Abdampfen etwas zu verändern und trocknen zu amorphen, rothgelb gefärbten, durchsichtigen Massen ein. Die Analyse der krystallwasserfreien Verbindung ergab Werthe, welche für ein Mononitrooxypyridin beweisend sind.

I. $0.2620 \mathrm{~g}$ Substanz gaben $0.4098 \mathrm{~g}$ Kohlensäure und $0.0709 \mathrm{~g}$ Wasser.

II. $0 \cdot 1428 g$ Substanz gaben $25 \cdot 3 \mathrm{~cm}^{3}$ Stickstoff bei $754 \cdot 8 \mathrm{~mm}$ und $18.6 \mathrm{C}$.

In 100 Theilen:

\begin{tabular}{cccc}
\multicolumn{1}{c}{ I } & II & $\mathrm{C}_{5} \mathrm{H}_{4} \mathrm{~N}_{2} \mathrm{O}_{3}$ \\
$\mathrm{C} \ldots \ldots \ldots \ldots$ & $42 \cdot 65$ & - & 42.85 \\
$\mathrm{H} \ldots \ldots \ldots \ldots$ & 3.00 & - & $2 \cdot 85$ \\
$\mathrm{~N} \ldots \ldots \ldots$ & - & $20 \cdot 26$ & 20.00
\end{tabular}

Was die Ausbeute an Nitroproducten betrifft, so haben wir bei Verarbeitung von $50 \mathrm{~g}$ Acetyloxypyridin circa $18 \mathrm{~g}$ reines Dinitro- $\beta$-Oxypyridin, neben $4 g$ des schwerlöslichen und $2 \cdot 5 \mathrm{~g}$ des leicht löslichen Mononitro- $\beta$-Oxypyridins erhalten.

Um grössere Mengen der Mononitro-Verbindungen zu gewinnen, haben wir eine Reihe von Versuchen ausgeführt, bei welchen wir geringere Mengen von Salpetersäure verwendeten und haben endlich nur jene Quantität derselben in Anwendung gebracht, die theoretisch zur Bildung von MononitroVerbindungen erforderlich waren. Der Erfolg hat jedoch bei all diesen Versuchen den Erwartungen nicht entsprochen. Es zeigte sich nämlich, dass in allen diesen Fällen ein grosser Theil des Acetyloxypyridins unverändert blieb, der nitrirte 
Antheil jedoch wieder in überwiegender Menge das Dinitroproduct enthält und nur äusserst geringe Quantitäten der beiden Mononitro-Verbindungen.

Auch die Änderung der Einwirkungstemperatur war nicht von bestimmendem Einfluss auf den Verlauf der Reaction. Es scheint daher, dass unter allen Umständen bei der Einwirkung der Salpetersäure auf die Acetylverbindung des Oxypyridins das Dinitrooxypyridin als Hauptproduct entsteht. 